\title{
Analizando la elección presidencial del 2012 desde la perspectiva de la agenda setting en la prensa de Monterrey
}

\author{
Emma Catalina GARZA AlANIS \\ Universidad de Monterrey (México) \\ caty.garzaa@gmail.com
}

Recibido: 08/11/2012

Aceptado: 23/01/2013

\begin{abstract}
Resumen
El presente estudio exploratorio prueba la teoría del agenda setting, se identificó si existe una relación entre la exposición a la agenda de la prensa escrita y la atención los lectores brindan a los temas establecidos por dicha agenda. Se utilizaron dos instrumentos (encuestas y análisis de contenido), y se encontró una correspondencia entre dos de los temas más mencionados en las encuestas y dos de los temas predominantes obtenidos mediante el análisis de contenido. Sin embargo la hipótesis del agenda setting de la prensa no se confirmó debido a que las personas manifestaron que el medio en que primero se informaron del tema (más importante) fue la televisión, se sugiere la necesidad de replantear el estudio desde la perspectiva de un establecimiento de la agenda compartido por lo medios.
\end{abstract}

Palabras clave: Agenda setting, prensa de Monterrey, Elección presidencial, Periodismo.

\section{Analyzing the Presidential Election of 2012 from the Perspective of Agenda Setting in Monterrey Press}

\begin{abstract}
This exploratory study tests the theory of agenda setting, it identifies if there is a relationship between exposure to the agenda of the press and the reader's attention to the themes established by that agenda. Two instruments were used (surveys and content analysis). A match between two of the most mentioned themes in surveys and two of the dominant themes obtained through content analysis were found. But the hypothesis of agenda setting of the press was not confirmed because people said that they were informed by television, this suggests the need to rethink the study from the perspective of a shared agenda setting by the media.
\end{abstract}

Key words: Agenda setting, Monterrey Press, Presidential election, Journalism.

\section{Referencia normalizada}

GARZA ALANIS, Emma Catalina (2013): “Analizando la elección presidencial del 2012 desde la perspectiva de la agenda setting en la prensa de Monterrey". Estudios sobre el mensaje periodístico. Vol. 19. Núm. especial abril, págs.: 793-801. Madrid, Servicio de Publicaciones de la Universidad Complutense.

Sumario: 1. Introducción; 1.1. Revisión de la literatura. 2. Metodología. 3. Desarrollo; 3.1. Encuestas; 3.2. Análisis de Contenido. 4. Conclusiones. 5. Referencias bibliográficas.

\section{Introducción}

La teoría del agenda setting no ha sido lo suficientemente explorada en México y con la intención de aportar en esta dirección, decidimos indagar si en el entorno de la elección presidencial 2012, los medios de comunicación establecen la agenda de lo que quieren que hable y piense la población, al elegir las historias que poseen interés informativo y decidir cuánto espacio e importancia se les darán. Debemos tomar en cuenta que a través de los medios las personas conocen a los candidatos y sus propuestas, debido a que difícilmente la población puede tener contacto directo con los 
aspirantes a la presidencia de México, por lo que adquiere gran valor el papel de los medios en las elecciones.

Las hipótesis que se manejaron en este estudio fueron las siguientes:

- H1: El electorado de Monterrey habla sobre temas electorales específicos manejados en la agenda de los medios debido a la exposición a la prensa escrita

- H2 El electorado de Monterrey habla sobre temas electorales específicos manejados en la agenda de los medios debido a la exposición a medios diferentes a la prensa escrita

- H3: El medio en que las personas se enteran primero de un tema o noticia, es diferente al medio en que prefieren informarse del mismo tema.

\subsection{Revisión de la literatura}

La teoría del agenda setting, establecimiento de la agenda, selección de temas o agenda de los medios son términos utilizados actualmente para referirse a una teoría sobre la relación de los medios y la opinión pública, que ha sido estudiada en múltiples ocasiones. La primera dimensión, confirmó que las personas hablan de los temas que los medios de comunicación les dan más espacio e importancia (López - Escobar, 1996). El presente trabajo se centró únicamente en la primera dimensión de la teoría.

Las raíces de la teoría del agenda setting se atribuyen a Walter Lippmann que en 1920 publicó Public Opinion. Después McCombs y Shaw (1972) tomaron la idea y realizaron un estudio en 1968 sobre las elecciones presidenciales de los Estados Unidos.

Algunos ejemplos de investigaciones y estudios realizados son las siguientes: Hay datos que señalan que la investigación de la agenda se ha extendido a otras áreas y una de ellas es la estudiada por Miller y Roberts (2010) quienes demostraron con los acontecimientos sucedidos el 11 de Septiembre del 2001 en la Zona Cero de Nueva York que el tamaño de una fotografía puede influenciar a un lector sobre la importancia de un tema. Esto ocurre porque las personas aceptan las imágenes y sus significados como representaciones de la realidad. Sus resultados fueron un posible efecto del agenda setting visual que abarca el uso de los medios con las imágenes.

El desarrollo de la teoría del establecimiento de la agenda llevó a Nguyen y Gehrau (2010) a señalar que una perspectiva integrada del agenda setting y de la comunicación interpersonal podría explicar porque en los estudios individuales se encuentran niveles bajos en los efectos de la agenda. Ellos propusieron un modelo de difusión de la agenda que integra la teoría two-step flow.

Uno de los estudios más recientes publicados relacionados con la política es el de Wirth, Matthes, Schemer, Wettstein, Friemel, Hanggli y Siegert (2010) que investigaron sobre la construcción y establecimiento de la agenda en campañas políticas y encontraron que los medios masivos de comunicación no tienen el mismo efecto directo y uniforme en todos los miembros de la audiencia.

Otro ejemplo de investigación sobre política en elecciones presidenciales es la de Wu y Coleman (2009) quienes realizaron un análisis de contenido y una encuesta de la elección presidencial del 2004 en Estados Unidos, en sus resultados encontraron que la percepción de los encuestados demostró el efecto de la agenda y que esto sirve para poder predecir la intención del voto. Los autores también afirmaron que después que 
surgió la teoría de la agenda, se descubrieron diversas condiciones con las cuales operaba la agenda, estas son "la necesidad de orientación" (need for orientation) y "la impertinencia de los temas" (obtrusiveness of issues).

Para Chernov (2011) "la necesidad de orientación" representa una condición clave para los efectos del agenda setting, define este concepto explicando que hay muchas situaciones donde los ciudadanos sienten que necesitan orientación para poder tomar decisiones, por ejemplo al momento del voto.

Aunque la teoría del agenda setting puede aplicarse en muchos campos, el área más fuerte de estudio ha sido la cuestión política. "Las plegarias, promesas y la retórica encapsulada en las nuevas historias, columnas y editoriales constituyen mucha de la información con la cual se realiza la decisión del voto. La mayoría de lo que la gente sabe proviene de 'segunda' o 'tercera' mano de los medios de comunicación o de las personas" (McCombs y Shaw, 1972: 176).

Según Paksoy (2010) la influencia del agenda setting no se puede restringir a los actos políticos, sino que la compleja estructura de la agenda posee las siguientes variables: Reglas y tradiciones del periodismo, interacciones diarias entre periodistas y compañías productoras de noticias, y el efecto de los periódicos en otros medios.

En la búsqueda realizada se encontraron tres estudios sobre la teoría del establecimiento de la agenda elaborados sobre las elecciones presidenciales del 2006 en México: Por medio de un análisis de contenido, McCombs y Valenzuela (2007) examinaron la influencia de la cobertura de noticias políticas sobre los candidatos presidenciales, en las cadenas televisivas Televisa y TV Azteca. Los resultados indicaron que ambas cadenas televisivas dieron una cobertura significativamente más amplia al candidato ganador Felipe Calderón Hinojosa, sobre sus rivales.

Sobre la segunda dimensión del agenda setting y el framing, Johnson, Davis y Cronin (2009) realizaron un análisis de contenido cuantitativo de 161 artículos y cualitativo de 36 artículos en la prensa estadounidense. Sus resultados fueron que Andrés Manuel López Obrador recibió mayor cobertura (59.6\%) que Roberto Madrazo Pintado (27.8\%) y Felipe Calderón Hinojosa (29.1\%).

Mediante el estudio a las elecciones presidenciales de México de 1994, 2000 y 2006, Espino (2009) concluyó que las estrategias de los medios de comunicación de los candidatos a la presidencia en el 2006 fue el factor más importante en la elección.

Podemos concluir que en ninguno de los estudios sobre la agenda setting en las elecciones presidenciales de México revisados se analizó al receptor, además parece ser que la comunicación es primordial para que un candidato pueda ganar las elecciones, Felipe Calderón Hinojosa tuvo mayor cobertura por lo medios y obtuvo el triunfo en las elecciones presidenciales del 2006 en México, mientras que en Estados Unidos, Andrés Manuel López Obrador recibió mayor cobertura.

Es importante conocer cuál es el consumo de medios en México para estudiar la teoría del agenda setting. Aguilar y Sierra (2010) refieren que el 60\% de los mexicanos leen el periódico (desde uno hasta los siete días en la semana), este porcentaje concuerda con la cifra de la Encuesta Nacional de hábitos, prácticas y consumo culturales de Conaculta (2010). 


\section{Metodología}

Para probar las hipótesis se aplicaron 200 encuestas "cara a cara" durante dos días a personas mayores de 18 años residentes del área metropolitana de Monterrey, Nuevo León. Esto con el propósito de obtener los temas que la muestra por conveniencia consideró más importantes sobre la elección presidencial.

También se realizó un análisis de contenido de la prensa escrita durante un día previo a la aplicación de las encuestas y los días de aplicación de la misma con el mismo propósito. Posteriormente se compararon los temas más importantes de las encuestas y del análisis de contenido.

Para este estudio sirvieron de guía las variables físicas que Acosta y Parra (1995) establecieron para el análisis y se añadieron otras, el análisis se compuso de: Fuente hemerográfica, ubicación, página par o non, tamaño (se incluirá la ilustración), ilustración, localización, tema, día y número de nota por fuente.

\section{Desarrollo}

Se obtuvieron los siguientes resultados repartidos en la sección de Encuestas y de Análisis de contenido.

\subsection{Encuestas}

La muestra constó de 112 Hombres (56\%) y 88 Mujeres (44\%) de 18 a 82 años, con un promedio de edad de 36 años. Al cuestionar a la muestra; ¿Cuál era el tema más importante sobre la elección presidencial 2012? en ese momento se obtuvieron las siguientes respuestas: "Visita de Andrés Manuel López Obrador y Enrique Peña Nieto al estado de Nuevo León" 47 personas (23\%), "campañas y propuestas" 46 personas (23\%), "Enrique Peña Nieto no cumple" 28 personas (14\%), "guerra sucia" 28 personas (14\%), "otros temas" 25 personas (12\%), "estadísticas y encuestas" 12 personas (6\%), "errores de Enrique Peña Nieto" 8 personas (4\%), "visita Josefina Vázquez Mota al estado de Nuevo León" 3 personas (2\%) y "debate de los candidatos" 3 personas $(2 \%)$.

La siguiente interrogante trató sobre el medio en que los encuestados se enteraron del tema que mencionaron era el más importante en ese momento sobre la elección presidencial 2012, se obtuvo que 90 personas (45\%) se enteraron por la "televisión", 44 personas (22\%) por "internet", 36 personas (18\%) Comentarios de amigos, familiares y/o conocidos, 22 personas (11\%) por el periódico y 8 personas $(4 \%)$ por la radio.

Al preguntar si las personas comentaron de manera personal o en redes sociales, el tema que mencionaron era el más importante la mayoría contestó que "si" 126 personas $(63 \%)$ y 74 personas (37\%) que "no".

A la muestra se le preguntó si necesitan obtener información previa para poder tomar una decisión sobre política, los resultados indicaron que: 166 personas (83\%) "totalmente de acuerdo", 20 personas (10\%) "de acuerdo", 9 personas (4\%) "totalmente en desacuerdo", 4 personas (2\%) "indiferente" y 1 personas $(0.5 \%)$ "desacuerdo".

Por último se cuestionó sobre el medio en el cuál los encuestados prefieren informarse sobre la elección presidencial, los resultados fueron los siguientes: El que más 
veces fue mencionado en primer lugar fue la "radio" con 53 personas (26\%), en segundo lugar "internet" con 50 personas $(25 \%)$, en tercer lugar "televisión" con 47 personas $(23 \%)$, en cuarto lugar "amigos, familiares y/o conocidos" con 27 personas (14\%) y en quinto lugar "prensa" con 23 personas $(12 \%)$.

\subsection{Análisis de contenido}

Se analizaron un total de 200 notas (artículos, caricaturas y/o comentarios de los lectores a los periódicos; todos publicados en las versiones impresas). Las publicaciones sumaron un total de $50,010.65 \mathrm{~cm} 2$.

El periódico con más notas sobre las elecciones presidenciales fue "Milenio" con 98 notas (49\%), seguido de "El Norte" con 65 notas (32.5\%) y de "El Porvenir" con 37 notas (18.5\%). En la variable de la ilustración se encontró que: 99 notas (49\%) “no poseían imagen".

Al analizar los temas a los que se les brindó más espacio y cantidad de notas se obtuvo: "campaña y propuestas" 20,490.80 cm2 (42\%), "visita de Andrés Manuel López Obrador y Enrique Peña Nieto al estado de Nuevo León" 11,913.10 cm2 (23\%), "elecciones $7217.64 \mathrm{~cm} 2$ (11\%), "otro" $6225.15 \mathrm{~cm} 2$ (11\%), "encuestas y estadísticas" $2219.15 \mathrm{~cm} 2$ (7\%) y "debate" $1944.05 \mathrm{~cm} 2$ (6\%).

Podemos apreciar en las siguientes tablas, una comparación entre las categorías que agrupan a los diversos temas que surgieron:

\begin{tabular}{|c|c|c|c|c|c|}
\hline & \multicolumn{2}{|l|}{ Encuestas } & & \multicolumn{2}{|c|}{ Análisis de Contenido } \\
\hline & Tema & Personas & & Tema & Notas $\left(\mathrm{cm}^{2}\right)$ \\
\hline 1 & Visitas AMLO y EPN & \begin{tabular}{|r|}
47 \\
\end{tabular} & 1 & Campañas y propuestas & $20,490.80$ \\
\hline 2 & Campañas y propuestas & 46 & 2 & Visitas AMLO y EPN & $11,913.10$ \\
\hline 3 & EPN no cumple & 28 & 3 & Elecciones & 7217.64 \\
\hline 4 & Guerra sucia & 28 & 4 & Otro & 6225.15 \\
\hline 5 & Otro & 25 & 5 & Encuestas y estadisticas & 2219.15 \\
\hline 6 & Encuestas y estadisticas & 12 & & Debate & 1944.05 \\
\hline 7 & Errores EPN & 8 & & & $50,010.70$ \\
\hline 8 & Visita JVM & 3 & & & \\
\hline & Debate & 3 & & & \\
\hline & & 200 & & & \\
\hline
\end{tabular}

Categorías de encuestas y del análisis de contenido (Elaboración propia)

\section{Conclusiones}

Para probar la primera dimensión de la teoría del agenda setting, la pregunta más importante de este estudio se centró en identificar si los temas que los electores consideraron que eran los más importantes en cuestión de la elección presidencial 2012 coincidieron con los temas que los medios establecieron como más relevantes (debido al espacio que se les brindó).

Al codificar los temas que los encuestados señalaron cómo más importantes se realizaron nueve categorías, los primeros dos temas que obtuvieron el mayor número de respuestas fueron: "Visita de Andrés Manuel López Obrador y de Enrique Peña Nieto al estado de Nuevo León" y el de "campañas y propuestas", los cuales fueron señalados por 93 personas (46.5\%), es decir casi la mitad de los encuestados. 
Parte importante de este trabajo fue comprobar si las personas comentan con otras personas los temas que establece la agenda, se comprobó que la mayoría de los encuestados, 126 personas (63\%), "sí" comentan los temas de la agenda, esto confirma lo que Nguyen y Gehrau (2010) propusieron, un modelo de difusión de la agenda que integra la teoría two-step flow. Utilizando la variable interviniente sexo en el hecho de que los encuestados si comentan el tema que definieron como más importante, se obtuvo que los hombres son los que comentan más y con esto se puede confirmar que podría haber en este caso un mayor efecto de la agenda en el sexo "masculino".

De las notas analizadas $49 \%$ no poseen imagen y $51 \%$ posee una fotografía a color, fotografía en blanco y negro o una caricatura. Miller y Roberts (2010) destacaron que las personas aceptan las imágenes y sus significados como representaciones de la realidad debido a la autoridad del medio que las respalda. Por lo tanto se presume que las imágenes que fueron publicadas en los periódicos sobre dos temas principales ("visita de Andrés Manuel López Obrador y Enrique Peña Nieto al estado de Nuevo León" y "Campañas y propuestas") pudieron influenciar a los encuestados estableciendo la agenda.

En los periódicos únicamente se publican temas actuales mientras que en las encuestas aparecieron temas antiguos como: "Errores de Enrique Peña Nieto", tema que ocupó espacio en los medios durante el mes de Diciembre, por lo que podría presumirse un efecto de persistencia del establecimiento de la agenda en los encuestados. Los encuestados también mencionaron temas como "Enrique Peña Nieto no cumple" y "guerra sucia" que no ocupan espacio en los diarios pero que son publicidad constante en los medios de comunicación.

Con respecto a la hipótesis uno: "El electorado de Monterrey habla sobre temas electorales específicos manejados en la agenda de los medios debido a la exposición a la prensa escrita" sí hay evidencia de un establecimiento del agenda debido a que cinco temas coincidieron entre las encuestas y el análisis de contenido, de los cuales dos ocupan las primeras posiciones con cerca de la mitad de las respuestas en las encuestas y más de la mitad del espacio publicado en los diarios. Sin embargo las personas manifestaron que el medio en que primero se enteraron del tema que señalaron fue la "televisión", por lo cual se presenta el efecto de la agenda pero no podemos atribuir a que se debe a la exposición a la prensa escrita. Sobre la hipótesis dos: "El electorado de Monterrey habla sobre temas electorales específicos manejados en la agenda de los medios debido a la exposición a medios diferentes a la prensa escrita" se acepta debido a que se sugiere que la agenda en este estudio fue establecida por la "televisión" y que esta agenda es influenciada por la agenda de los periódicos. Por lo tanto, se comprobó la presencia de la primera dimensión de la teoría del agenda setting, y se confirmó que las personas hablan de los temas a los que los medios de comunicación les dan más espacio e importancia (López - Escobar, 1996).

Sobre la preferencia de medios en el primer lugar, para la muestra el que más veces fue mencionado en primer lugar fue la "radio", en segundo lugar la "prensa, en tercer lugar los "comentarios de amigos, conocidos y/o familiares". Se continúo tomando la variable de sexo en el análisis de la preferencia del primer lugar y se obtuvo que las mujeres eligieron el Internet, y los hombres la Radio. Con la variable del estrato so- 
cioeconómico se obtuvo que la clase Baja prefiere la Radio, la Media el Internet y la Alta la Radio. Por último, con la variable del grado máximo de estudios las personas Con estudios universitarios (con carrera profesional, maestría y/o doctorado terminado) y las personas Sin estudios universitarios (con primaria, secundaria y/o bachillerato terminado) prefieren la Radio.

Sobre la tercera hipótesis "El medio en que las personas se enteran primero de un tema o noticia, es diferente al medio en que prefieren informarse del mismo tema" se acepta debido a que se encontró en el estudio que las personas se enteraron de la noticia por la "televisión" pero prefieren enterarse sobre las elecciones en la "radio" que ocupó el primer lugar en las preferencias o la "prensa" que ocupó el segundo. Esto pudiera deberse a que la "radio" y la "prensa" poseen más credibilidad que la "televisión", pero esta última es más accesible. Los resultados de la Encuesta Nacional de hábitos, prácticas y consumo culturales de Conaculta en Nuevo León (2010) revelaron que un $73.7 \%$ lee el periódico, $74 \%$ escucha la radio, mientras que nuestro estado $94 \%$ de los neoloneses ve la televisión (cada porcentaje fue calculado sobre $100 \%$ ), es evidente el mayor consumo de la "televisión", a esto puede deberse los resultados obtenidos en el presente estudio.

Al cuestionar a la muestra sobre la necesidad de obtener información previa para tomar sus decisiones políticas se encontró que la mayoría está de acuerdo 186 (93\%) personas, mientras que sólo $13(0.65 \%)$ están en desacuerdo y $1(0.5 \%)$ es indiferente. Para Chernov (2011) "la necesidad de orientación" representa una condición clave para los efectos del agenda setting, se puede confirmar que los señalamientos de Chernov se comprobaron en la investigación.

Aunque el presente estudio es cuantitativo, al encuestar surgió que el tema que las personas mencionaban como más importante estaba relacionado con la intención del voto, además aunque no se tomó nota de los comentarios cualitativos de los encuestados, era un agregado que las personas manifestaban por quién iban a votar y por lo tanto hay evidencia que ponen más atención en las noticias que refuerzan su decisión, esto fue señalado por Joseph Thomas Klapper al definir los efectos limitados de los medios, entendiendo que éstos más que crear o modificar valores, refuerzan los existentes (Infoamérica, 2011).

Los resultados obtenidos en esta investigación fueron son reveladores pero sin duda sería necesario tener más tiempo para profundizar en la investigación, realizar un análisis de contenido en los demás medios de comunicación y aplicar un plan de muestreo representativo. El presente trabajo es una investigación exploratoria que pretende marcar direcciones para investigaciones futuras que indaguen más sobre la discrepancia entre el medio por el que las personas se enteran de temas políticos y el medio que prefieren para informarse.

Para finalizar considero que el efecto del agenda setting siempre estuvo presente en los encuestados sin importar su sexo, nivel socioeconómico y grado máximo de estudios; y también que la agenda de los medios es compartida por todos éstos, conformando un establecimiento de la agenda integral. 


\section{Referencias bibliográficas}

ACOSTA; Miguel y PARRA; Luz Paula (1995): Los procesos electorales en los medios de comunicación. Guía para el análisis de contenido electoral en México. México, Universidad Iberoamericana.

AGUILAR, Yanet y SIERRA, Sonia (2010): "El mexicano lee poco, no va a museos y ve mucha tv", en El Universal: http://www.eluniversal.com.mx/cultura/64416.html [fecha de consulta: 16 de febrero de 2012].

CHERNOV, Gennady; VALENZUELA, Sebastián y MCCOMBS, Maxwell (2011): "An experimental comparison of two perspectives on the concept of need for orientation in agenda-setting theory". Journalism and Mass Communication Quarterly, no 88. USA, Sage Journals, pp. 142-155.

CONACULTA (2010): “Encuesta Nacional de hábitos, prácticas y consumo culturales", en http://www.conaculta.gob.mx/recursos/banners/ENCUESTA_NACIONAL.pdf. [fecha de consulta: 17 de febrero de 2012].

ESPINO, Germán (2009): "The transformation of political communication in Mexico (1994 - 2006)". "The transformation of political communication in Mexico (19942006)". Journal of Global Communication, $\mathrm{n}^{\circ}$ 2. India, Indian Journals, pp. 225247.

INFOAMÉRICA (2011): “Joseph Thomas Klapper”, en Infoamérica: http://www.infoamerica.org/teoria/klapper1.htm. [fecha de consulta: 15 de mayo de 2012].

JOHNSON, Melissa; DAVIS, John, y CRONIN, Sean (2009): "Mexican expatriates vote? Framing and agenda setting in U.S. news coverage about Mexico". Mass Communication \& Society, $\mathrm{n}^{\mathrm{o}}$ 12. USA, AEJMC, pp. 4-25.

LIPPMANN, Walter (1920): Public Opinion. New York, Macmillan.

LÓPEZ-ESCOBAR, Esteban (1996): “Agenda-setting: investigaciones sobre el primero y el segundo nivel" en Comunicación y Sociedad: http://www.unav.es/fcom /comunicacionysociedad/es/articulo.php?art_id=286. [fecha de consulta: $15 \mathrm{de}$ febrero del 2012].

MCCOMBS, Maxwell y SHAW, Donald (1972): "The agenda setting function of the mass media". Public Opinion Quarterly, $\mathrm{n}^{\circ} 36$. UK, Oxford University Press, pp. 176-187.

MCCOMBS, Maxwell y VALENZUELA, Sebastián (2007): “Agenda-setting effects on vote choice: Evidence from the 2006 Mexican election". Communication \& Mass Media Complete. USA, EBSCO HOST Publishing, pp. 1-45.

MILLER, Andrea, y ROBERTS, Sharon (2010): "Visual agenda-setting and proximity after hurricane Katrina: A study of those closest to the Event". Visual Communication Quarterly, $\mathrm{n}^{\circ}$ 17. USA, Rutledge, pp. 31-46.

NGUYEN, Hong y GEHRAU, Walker (2010): "Agenda diffusion: An integrated model of agenda setting and interpersonal communication". Journalism and Mass Communication Quarterly, $\mathrm{n}^{\circ}$ 87. USA, Sage Journals, pp. 100-116. 
PAKSOY, Aladdin. (2010): “Deceleration of Turkey's EU accession on media agenda: A case study on the guardian coverage between 2003 and 2010". Communication \& Mass Media Complete, $\mathrm{n}^{\circ}$ 14. USA, EBSCO HOST Publishing, pp. 37-54.

TAN, Yue y WEAVER, David (2010): "Media bias, public opinión and policy liberalism from 1956 to 2004: A second-level agenda-setting study. Mass Communication and Society", n ${ }^{\circ} 13$. USA, AEJMC pp. 412-434.

WIRTH, Werner; MATTHES, Jörg; SCHEMER, Christian; WETTSTEIN, Martin; FRIEMEL, Thomas; HÄNGGLI, Regula; y SIEGERT, Gabriele (2010): “Agenda building and setting in a referendum campaign: Investigate the flow of arguments among campaigners, the media, and the public". Journalism and Mass Communication Quarterly, n ${ }^{\circ}$ 87. USA, Sage Journals, pp. 328-345.

WU, H. Denis y COLEMAN, Renita (2009): Advancing agenda-setting theory: The comparative strength and new contingent conditions of the two levels of agendasetting effect". Journalism and Mass Communication Quarterly, $\mathrm{n}^{\circ}$ 86. USA, Sage Journals, pp. 775-789.

\section{Emma Catalina GARZA ALANIS}

Universidad de Monterrey

Licenciada en Ciencias de la Información y Comunicación.

caty.garzaa@gmail.com 